\section{The Breakup of a Main-Belt Asteroid 450 Thousand Years Ago}

\author{
David Nesvorný, ${ }^{1 *}$ David Vokrouhlický, ${ }^{1}$ William F. Bottke ${ }^{1}$
}

$\mathrm{W}$ e selected 264,403 asteroids from the Lowell Observatory catalog $(1)$ that have observational arcs longer than 10 days, and we used the hierarchical clustering method (HCM) (2) to search for groups. The HCM selects groups of orbits in (semimajor axis $a$, eccentricity $e$, inclination $i$, perihelion longitude $\varpi$, nodal longitude $\Omega$ ) space based on distances between two neighbor orbits. We used the standard definition of distance in $(a, e, i)$ space, various definitions of distance in $(\varpi, \Omega)$ space, and different cut-off values to test the sensitivity of the method on these parameters. One new and two previously known asteroid families were found. The Iannini and Karin clusters, known to be 1 to 5 million years (My) old and $\approx 5.8$ My old $(3,4)$, respectively, showed up as several groups with five to ten members, each representing a small part of the two families that has maintained a coherent distribution of osculating orbits until the present epoch.

The newly identified family is a group of six 1- to 2.5-km-diameter asteroids, whose orbits are very tightly clustered near the inner main belt of the $\approx 10$-km-diameter object 1270 Datura (Table 1). It is extremely unlikely that such a concentration of orbits is a random fluctuation. The small dispersion of orbits in $a, e$, and $i$ indicates velocity perturbations $\delta V \approx 2 \mathrm{~m} \mathrm{~s}^{-1}$ relative to Datura, except for (89309) 2001VN36, which has $\delta V \approx 5 \mathrm{~m} \mathrm{~s}^{-1}$. These values are comparable to the expected escape speed from a 10-km-diameter asteroid $\left(\approx 5 \mathrm{~m} \mathrm{~s}^{-1}\right)$

Table 1. Values of $\varpi$ and $\Omega$ for asteroids in the Datura cluster on mean Julian date (MJD) 2453700.5. Time-averaged (proper) orbital elements of 1270 Datura are $a=2.23468 \mathrm{AU}, e=0.15341$, and $\sin (i)=0.09230$. All remaining members except (89309) 2001 VN36 have the average orbital elements within $3 \times 10^{-4} \mathrm{AU}, 10^{-4}$, and $10^{-4}$ to these values, respectively. (89309) 2001 VN36 has a displacement in $a, e$, and $\sin (i)$ that is $\approx 3$ times larger. All members of the Datura cluster are tightly clustered in $\varpi$ and $\Omega$. Absolute magnitudes $H$ were taken from (1).

\begin{tabular}{llccc}
\multicolumn{1}{c}{ Asteroid } & $\begin{array}{c}\boldsymbol{H} \\
(\mathrm{mag})\end{array}$ & $\begin{array}{c}\varpi \\
\left({ }^{\circ}\right)\end{array}$ & $\begin{array}{c}\boldsymbol{\Omega} \\
\left({ }^{\circ}\right)\end{array}$ \\
\hline$(1270)$ & Datura & 12.5 & 356.58 & 97.90 \\
$(60151)$ & 1999UZ6 & 16.3 & 357.58 & 96.80 \\
$(89309)$ & 2001VN36 & 16.3 & 359.78 & 93.00 \\
$(90265)$ & 2003CL5 & 15.4 & 357.71 & 95.70 \\
& 2001WY35 & 17.0 & 357.29 & 96.89 \\
& 2003SQ168 & 16.9 & 356.72 & 97.49 \\
& 2003UD112 & 17.9 & 358.62 & 95.47 \\
\hline
\end{tabular}

and are much smaller than typical asteroid orbital speeds at 2.235 astronomical units (AU) $(\approx 20 \mathrm{~km}$ $\mathrm{s}^{-1}$ ). Moreover, the size frequency distribution of Datura cluster members, which include one large and numerous small asteroids, is also characteristic of impact-generated debris. Given these results, we propose that the Datura cluster is the remnant of a larger parent asteroid that was disrupted by a collision with a $\approx \mathrm{km}$-sized asteroid.

To determine the age of the Datura cluster, $t_{\text {age }}$, we numerically tracked the present orbits of four Datura cluster members backward in time. We did not use (89309) 2001VN36, which is strongly chaotic due to effects of the 9:16 orbital resonance with Mars, nor did we use 2003 SQ168 and 2003 UD112, which have large orbital uncertainties. A total of 840 alternative orbit histories

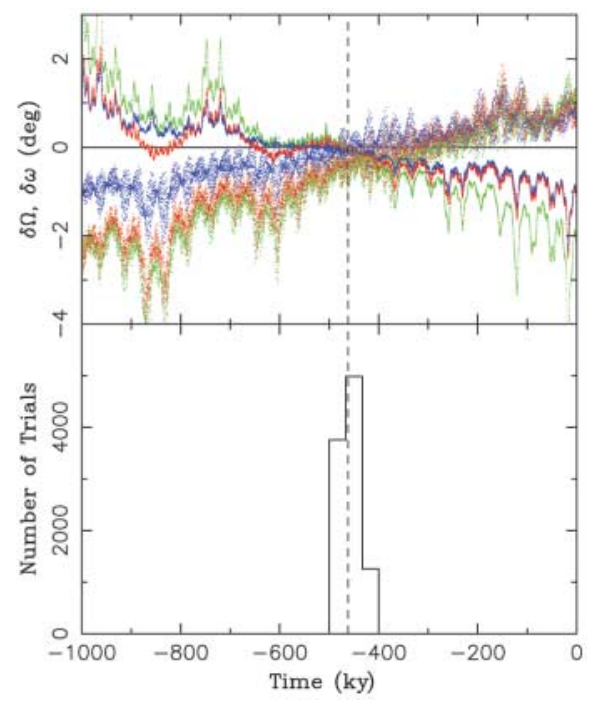

Fig. 1. Convergence of orbits suggests that the Datura cluster formed $450 \pm 50 \mathrm{ky}$ ago. (Top) Past evolutions of $\varpi$ (ascending lines) and $\Omega$ (descending lines) for (60151) 1999 UZ6 (red), (90265) 2003CL5 (blue), and 2001WY35 (green). Values relative to 1270 Datura are shown. The dashed vertical line denotes time $t=-467 \mathrm{ky}$, when smallest dispersion of $\varpi$ and $\Omega$ occurred for this trial. Here we used semimajor axis drift rates daldt $=-2.8 \times 10^{-5}$, $-2.3 \times 10^{-4}, 1.5 \times 10^{-5}$, and $-6.2 \times 10^{-5} \mathrm{AU}$ $\mathrm{My}^{-1}$ for 1262 Datura, (60151) 1999UZ6, (90265) 2003 CL5, and 2001WY35, respectively. These values are within the range of daldt suggested by the theory of the Yarkovsky effect (5). (Bottom) The histogram of plausible $t_{\text {age }}$ determined from $10^{7}$ different orbital histories of Datura cluster members. Plotted here are all $t_{\text {age }}$ for which $\delta V<5 \mathrm{~m} \mathrm{~s}^{-1}$. were produced for each of the four asteroids that differed by the starting orbit (chosen randomly within the orbit uncertainty range for each asteroid) and magnitude of Yarkovsky thermal drag (5). We randomly selected one orbital history for each Datura member asteroid and determined $t_{\text {age }}$ for this trial by requiring that the dispersion in $\varpi$ and $\Omega$ at $t_{\text {age }}$ corresponds to $\delta V<5 \mathrm{~m} \mathrm{~s}^{-1}$. Such a convergence is expected at the time of the breakup. For comparison, the dispersion of $\varpi$ and $\Omega$ at the current epoch corresponds to $\delta V \approx 40 \mathrm{~m} \mathrm{~s}^{-1}$. The range of plausible $t_{\text {age }}$ values was determined from $10^{7}$ trials. The result shows that the Datura cluster is $450 \pm 50$ thousand years (ky) old (Fig. 1), considerably younger than other known asteroid families.

Existing color data indicate that 1270 Datura has a taxonomic type within the asteroidal S complex, which is thought to be compositionally related to the ordinary chondrite meteorites. 1270 Datura has a short, $\approx 3.3$ hours rotation period, possibly as a result of momentum transfer occurring during the family-forming collision. Based on impact simulations with a hydrodynamic code, we estimate that the disrupted parent body was $\approx 15 \mathrm{~km}$ in diameter. Apparently, a substantial fraction of the parent body's mass was ejected to space as fragments ranging in size down to micrometer-sized dust particles.

The production of these particles implies that the Datura cluster may be a source of some of the material in the circumsolar (zodiacal) dust cloud. Based on the Datura cluster's inclination $\left(\approx 5.3^{\circ}\right)$, we speculate that it might be the source for the $\mathrm{E} / \mathrm{F}$ dust band pair discovered by the infrared astronomical satellite (IRAS) in 1983 (6). We estimate that micrometer-sized Datura particles migrate by radiation effects from $2.235 \mathrm{AU}$ to $1 \mathrm{AU}$ in only $\approx 2000$ years. Therefore, a wave of micrometersized Datura particles may have reached Earth only a few thousand years after the formation of the Datura cluster. Signs of this event may be found by analyzing tracers of extraterrestrial dust in deep ocean sediments and Antarctic ice cores.

References and Notes

1. E. Bowell, K. Muinonen, L. H. Wasserman, in Asteroids, Comets and Meteors, A. Milani et al., Eds. (Kluwer, Dordrecht, 1994), pp. 477-481.

2. V. Zappalà, A. Cellino, P. Farinella, Z. Knežević, Astron. J. 100, 2030 (1990).

3. D. Nesvorný, W. F. Bottke, H. F. Levison, L. Dones, Nature 417, 720 (2002).

4. D. Nesvorný, W. F. Bottke, H. F. Levison, L. Dones, Astrophys. J. 591, 486 (2003).

5. W. F. Bottke, D. Vokrouhlický, D. P. Rubincam, M. Broz, in Asteroids III, W. F. Bottke, A. Cellino, P. Paolicchi, R. Binzel, Eds. (Univ. of Arizona Press, Tucson, 2002), pp. 395-408. 6. M. V. Sykes, Astrophys. J. 334, L55 (1988).

7. This manuscript is based upon work supported by NASA's Planetary Geology and Geophysics program and the Czech Grant Agency.

13 February 2006; accepted 3 April 2006 10.1126/science. 1126175

${ }^{1}$ Department of Space Studies, Southwest Research Institute, 1050 Walnut Street, Suite 400, Boulder, CO 80302, USA.

*To whom correspondence should be addressed. E-mail: davidn@boulder.swri.edu 